\title{
Calidad de los servicios de Salud Pública en San
}

\section{Salvador}

José Ricardo Gutiérrez Quintanilla

Investigador, Dirección de investigaciones de la UTEC

\section{Resumen}

El presente estudio tenía como objetivo principal hacer una evaluación de la calidad de atención en los servicios de salud en la ciudad de San Salvador. Para ello, se diseñó un instrumento a partir del modelo teórico sugerido por Donabedian (1966), que contiene las dimensiones de estructura, proceso-resultado y accesibilidad. Es un estudio descriptivo mediante encuesta (León y Montero, 2007), su diseño retrospectivo; la muestra es no probabilística de tipo incidental, constituida por 1,210 personas entre hombres $(42,1 \%)$ y mujeres $(57,9 \%)$ con edad media de 32,5 años. Los resultados muestran que el cuestionario (ECASS) posee adecuadas propiedades psicométricas de fiabilidad y validez; asimismo, se encontró que existen diferencias significativas en la calidad de atención en salud, entre los centros de atención privada y los públicos, indicando que es de mejor calidad la atención en los centros privados. Algunos indicadores considerados como negativos: mala atención en el sistema de salud público salvadoreño $(63,4 \%)$; los tiempos de espera entre una cita y otra son muy largos; $(70,8 \%)$, en el sistema de salud pública, el acceso al médico especialista es sumamente difícil $(68,4 \%)$. Los resultados pueden servir de parámetros para que a partir de ellos, se implementen mejoras en el proceso de atención en salud pública.

\section{Palabras claves:}

Calidad, atención en salud, fiabilidad, validez.

\section{Introducción}

1 a evaluación de los servicios de salud inicia en los EE.UU., en la década de los 50 del siglo XX. Comienza con la acreditación de calidad de atención en los hospitales. En este contexto surgen, muchas propuestas en diferentes estados. Ejemplo: la de Chicago, Washington, la escuela de salud publica de llinois. Todas ellas centradas en la construcción de estándares para evaluar la calidad de los servicios de salud; de esta forma, aparece el proyecto (Oliver, 2005) nacional de medición de estándares y desempeño de la Salud
Pública de EE.UU. por sus siglas en inglés (NPHPSP), el cual establece cuatro niveles para medir la calidad de atención en salud publica: a) cumplimiento entre el 75 y el $100 \%$ ) = Sí. b) Cumplimiento entre el 51 y el $75 \%$ $=$ parcialmente alto. c) Cumplimiento entre el 26 y el $50 \%$ ) = parcialmente bajo, y d) Cumplimento $<25 \%$ ) $=$ "No. Facilitando herramientas normalizadas para medir la calidad de la atención en salud pública.

En América Latina, es a partir de los 70 cuando surgen algunos esfuerzos limitados especialmente en Brasil,
Argentina y Colombia, los cuales no han tenido mayor importancia; en EI Salvador, se desarrolló un proyecto de programa nacional de gestión de evaluación de calidad en los servicios de salud, para ser implementado en el quinquenio 2004-2009, el cual nunca se concretizó.

En la actualidad, existe una percepción bastante generalizada de la mala calidad de los servicios de salud pública en El Salvador; reflejándose en el autoestudio cualitativo del MSPAS (2005), el cual enfatiza en los problemas en la 


\begin{abstract}
This study had as main objective to assess the quality of care in health services in the city of San Salvador. To this end, an instrument was designed from the theoretical model suggested by Donabedian (1966), which contains the dimensions of structure, process-outcome and accessibility. It is a descriptive survey study (León and Montero, 2007), its design is retrospective and the sample is a non probabilistic incidental type, consisting of 1.210 persons, males $(42.1 \%)$ and females $(57.9 \%)$ with mean age of 32.5 years. The results show that the questionnaire (ECASS) has adequate psychometric properties of reliability and validity, also found significant differences in the quality of health care among private health care centers and public, indicating that it is of better quality care in private hospitals. Some indicators considered negative: poor attention in the Salvadoran public health system $(63.4 \%)$, the waiting time between an appointment and another is very long; $(70.8 \%)$ in the public health system, the access to a medical specialist is extremely difficult $(68.4 \%)$. The results can serve as benchmarks for that from them, implement process improvements in public health care.
\end{abstract}

\section{Key words:}

Quality, healthcare, reliability, validity

suficiencia y preparación del recurso humano, deficiencias en la gestión de insumos asistenciales, deficiencias en monitoreo y supervisión, falta de estandarización en la prestación de los servicios de salud, ausencia de un sistema de categorización. En este mismo informe, se confirma la mayoría de los problemas relacionados con la calidad de los servicios de salud del Sistema Nacional, con la diferencia de que estas han sido señaladas por profesionales externos al sistema. Se tienen las siguientes: deficiencias en la infraestructura física y en la dotación de recursos humanos en el área asistencial, falta de oportunidad en los servicios, inequidades en el acceso a la tecnología, escasa coordinación entre los formadores del talento humano y los proveedores de servicios de salud, limitada coordinación interinstitucional e intersectorial, atraso tecnológico que dificulta la atención de los usuarios y la gestión de la información, entre otras.
El propósito general del presente estudio fue evaluar la calidad de atención en los servicios de salud pública de San Salvador, incluyendo en este indicadores de infraestructuras: condiciones de comodidad del paciente, distribución fisica y mantenimiento del centro de salud; proceso-resultado: agilidad en los procesos administrativos, cómo sacar una cita, el expediente clínico, resultado de exámenes (laboratorio y Rx), atención del paciente, tanto del médico como de la enfermera, evaluación y tratamiento medico recibido, efectividad del tratamiento y accesibilidad: acceso al sistema de salud, al médico general y especialista, los tiempos de espera entre cita y cita y el día de la consulta.

En los últimos años, se han realizados diferentes esfuerzos desde los estados orientados a reorganizar los sistemas de salud en la mayoria de paises latinoamericanos, con el propósito de brindar servicios de salud de calidad a la población. EI Salvador no ha sido la excepción, (ejemplo: Sistema Integral de Salud). Estos esfuerzos han derivado en múltiples conflictos de intereses entre las autoridades de salud y los sindicatos de trabajados de la salud. Estas crisis (Ej: paros laborales, marchas) han obedecido a diferentes causas, como son: exigencias salariales, prestaciones, incremento del presupuesto en salud. Uno de

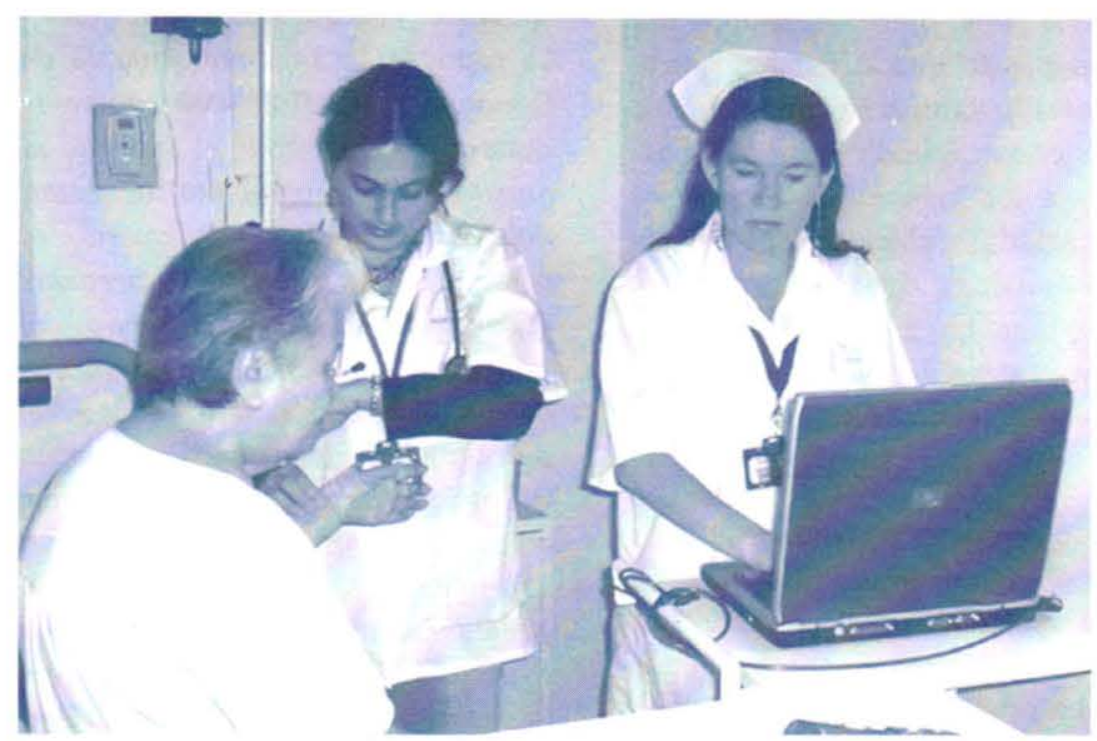




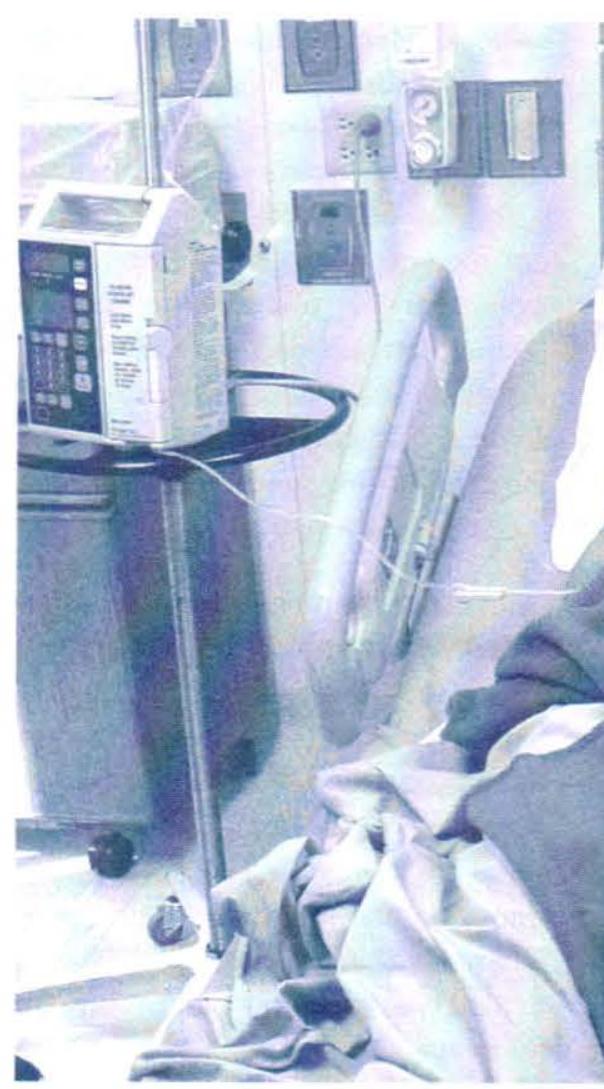

los mayores conflictos ha sido la luchas de los profesionales de la salud para evitar el componente privatizador (dogma neoliberal) de algunos servicios de salud (limpieza, lavandería, alimentación, seguridad, cirugías e infraestructura), que históricamente fueron prestados por empleados delEstado. Estas crisis han desgastado tanto al sistema de salud (Ministerio de Salud y Seguro Social), como a los mismos profesionales de la salud, incidiendo en la calidad de los servicios de salud prestados; esto último ha configurando en no pocos salvadoreños una imagen relativamente desfavorable hacia el sistema de salud salvadoreño.

En lo referente al sector salud, se han difundido varias definiciones sobre calidad en los servicios, entre las más relevantes se tienen dos. La primera: "Es el desempeño

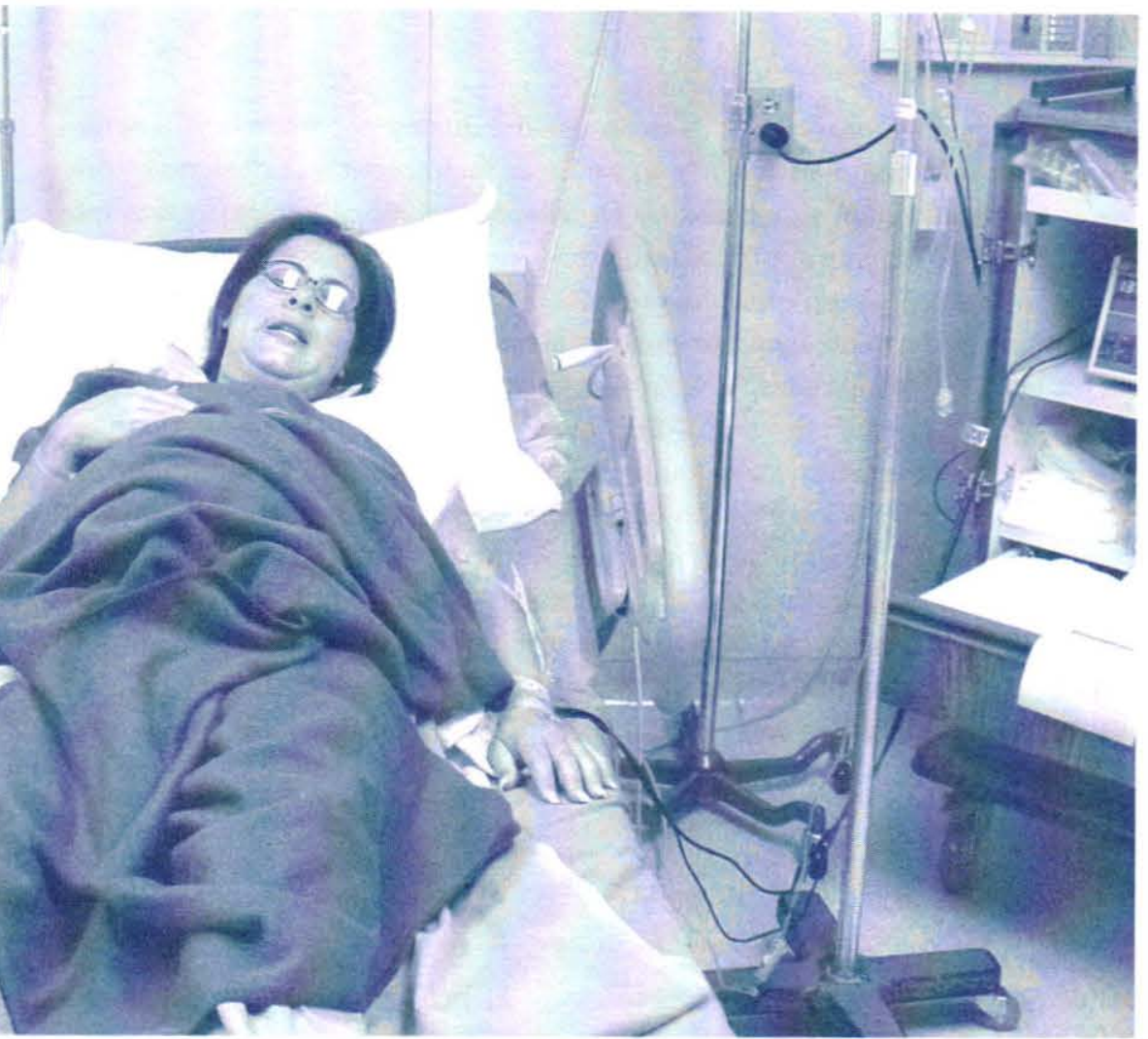

apropiado (acorde con las normas) de las intervenciones que se saben son seguras, que la sociedad en cuestión puede costear y que tiene la capacidad de producir un impacto, sobre la mortalidad, la morbilidad, la incapacidad y la desnutrición." (Roemer y Montoya, OMS; 1988). La segunda: la más conocida, la de Donabedian, (1974, 1988) quien la define: "Proporcionar al paciente el máximo y más completo bienestar, después de haber considerado el balance de las ganancias y pérdidas esperadas, que acompañan el proceso de atención en todas sus partes". Esta calidad recoge el pensamiento técnico de quienes la aplican (médicos, enfermeras, administradores) con su cosmovisión y formación académica (Donabedian, 1984). Existe consenso entre los especialistas de que Donabedian es la referencia obligada para tratar, evaluar, estudiar la calidad de la atención en los servicios de salud, debido a que es el autor que más ha investigado y escrito sobre el tema.

\section{Método}

\section{Participantes}

La muestra estaba formada por 1,210 personas entre hombre y mujeres de la población general de San Salvador. El tamaño de la muestra obtenido tiene un nivel de confianza del $95 \%$ con un error de estimación del $3 \%$. Es un estudio descriptivo mediante encuesta (León y Montero, 2007), con un diseño retrospectivo; el muestreo es no probabilístico de tipo incidental (Hernández, Fernández, y Baptista 2006). Sin embargo, destacar que la selección del número de participantes se obtuvo mediante 
la fórmula para poblaciones finitas, apoyando la posibilidad de obtener generalizaciones de sus hallazgos, mediante la utilización de pruebas paramétricas y no paramétricas, en el análisis de resultados. De esta muestra, $688(57,9 \%)$ fueron femeninos con una media de edad de 32,0 años (DT $=12,0)$, mientras $501(42,1 \%)$ fueron masculinos con una media de edad de 33,2 años (DT $=12,5)$; la media de edad general fue de 32,5 años (DT=12,23). La muestra estudiada residía en el área metropolitana de San Salvador. Las variables sociodemográficas como institución de salud que visita, el género, estado familiar, nivel de estudio, número de miembros en el hogar e ingresos económicos familiares.

\section{Instrumentos}

Instrumento de elaboración propia.

Se elaboró un cuestionario que buscaba medir un conjunto de variables sociodemográficas consideradas fundamentales para el estudio; entre estas se tienen: la edad, institución de salud que visita, el género, estado familiar, nivel de estudio, número de miembros en el hogar e ingresos económicos familiares. Variables que fueron utilizadas para realizar los análisis estadísticos inferenciales, tanto paramétricos como no paramétricos.

Escala de calidad de atención en los servicios de salud (ECASS).

Tras la revisión de algunos modelos teóricos sobre la calidad de atención en los servicios de salud, se adoptó el modelo de Donabedian (1966), quien plantea que la calidad de atención en salud deberá evaluarse desde la perspectiva del usuario y medirse cuatro fases importantes: estructura, proceso, resultados y accesibilidad y que, en su conjunto, brindarían un puntaje o criterio sobre la calidad de atención en salud; sin embargo, podrán hacerse mediciones parcializadas de algunas de las fases o etapas del proceso de evaluación, según las necesidades o propósitos del estudio. En esta ocasión, ante la inexistencia de instrumentos válidos y fiables que evaluaran la calidad de atención en los servicios de salud pública, se procedió a operativizar la estructura propuesta por el autor (estructura, proceso, resultado y accesibilidad) de cara a la construcción de un instrumento atendiendo los estándares internacionales.

\section{Resultados}

Tras un proceso de recodificación de las variables (ítems) de un nivel de medición de intervalo ( $1=$ mala, $2=$ regular, 3 =buena, $4=$ muy buena) a un nivel dicotómico $(1+2=$ mala/os; $3+4=$ buena/os) de mala/os y buena/ os, para cada reactivo se obtuvo (n) el total de la muestra, la frecuencia y porcentaje tanto para las respuestas malas como para las buenas. En el análisis descriptivo de la calidad de atención en los servicios de salud, se ha utilizado como criterio de interpretación de los hallazgos descriptivos, la norma del proyecto (Oliver, 2005) Nacional de Medición de Estándares y Desempeño de la Salud Pública de EE.UU, por sus siglas en inglés (NPHPSP), el que establece cuatro niveles para medir la calidad de atención en salud pública; estos son:1) cumplimiento entre el 75 y el $100 \%$ )=Sí. 2) Cumplimiento entre el 51 y el $75 \%=$ parcialmente alto. 3) Cumplimiento entre el 26 y el $50 \%=$ parcialmente bajo, y 4) Cumplimiento menor al $25 \%$ ) = "No. Es importante aclarar que en nuestra escala el malo es equivalente al "No" y el bueno es equivalente al "Si". Considerando los parámetros anteriores para valorar aquellos indicadores estudiados como buenos o malos, se procedió entonces a describir los indicadores

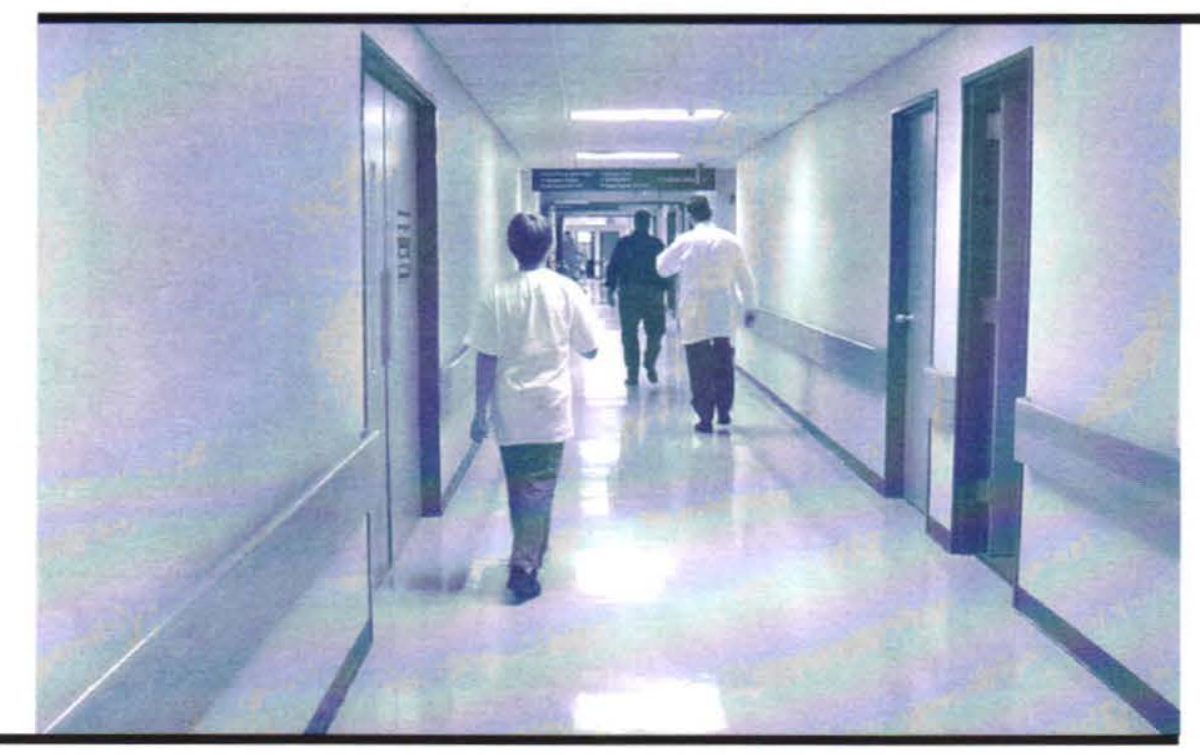


En los criterios donde el porcentaje malo es inferior al $\mathbf{5 0} \%$, es considerado como desfavorable o negativo (parcialmente bajo), mientras en aquellos indicadores cuyo resultado es bueno, serán los que están arriba del $51 \%$.

cuyos porcentajes oscilaron entre 26 a $50 \%$ como parcialmente bajos y los que oscilaron entre 51 a $75 \%$ como parcialmente altos. No se encontraron porcentajes superiores al $76 \%$ que significan "si". Tampoco inferiores al $25 \%$ que significa un "no". En los criterios donde el porcentaje malo es inferior al $50 \%$, es considerado como desfavorable o negativo (parcialmente bajo), mientras en aquellos indicadores cuyo resultado es bueno, serán los que están arriba del $51 \%$. Estos últimos son valorados como positivos (parcialmente altos). En la breve descripción de estos resultados, se está haciendo énfasis en los seis indicadores o porcentajes más elevados, tanto en la percepción positiva como en la negativa de la calidad de atención en los servicios de salud pública. En primer lugar, tenemos algunos indicadores más llamativos considerados como desfavorables o malos: la atención que recibe de la enfermera/os $(57,2 \%)$; los procesos administrativos como sacar una cita, solicitar una constancia médica $(59,8 \%)$; la atención en el sistema de salud público salvadoreño $(63,4 \%)$; los tiempos de espera entre una cita y otra $(70,8 \%)$; cuando se presenta a consulta el tiempo de espera para que lo

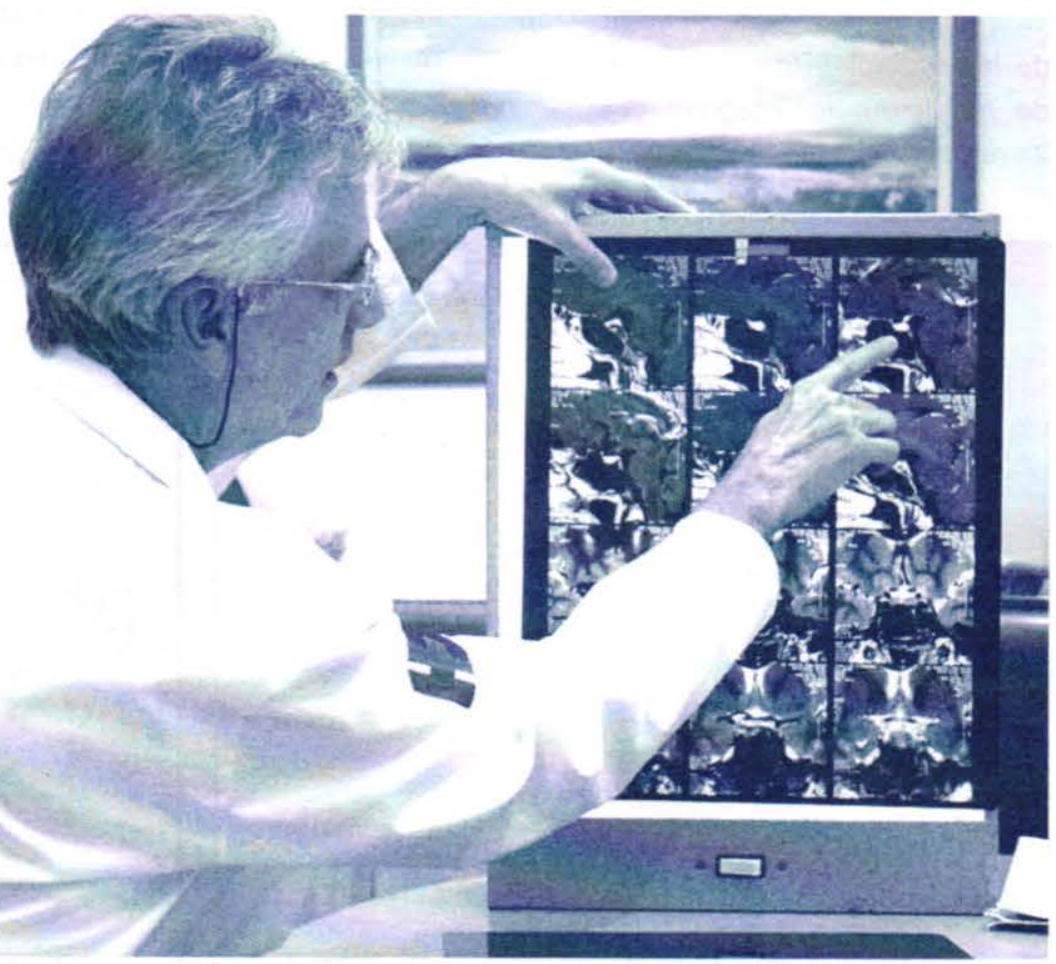

vea el médico $(69,0 \%)$; en el sistema de salud público, el acceso al médico especialista $(68,4 \%)$. En segundo lugar, tenemos algunos indicadores más sobresalientes considerados como buenos: el centro de salud está limpio y ordenado $(64,3 \%)$; la evaluación diagnóstica y el tratamiento médico recibido $(57,8 \%)$; ha sentido mejoría en su estado de salud después de iniciado el tratamiento $(63,9 \%)$; el tratamiento médico recibido lo percibe como efectivo en curar la enfermedad $(59,7 \%)$; la explicación del médico sobre su enfermedad fue con claridad $(57,1 \%)$; y la accesibilidad (por distancia) a un centro de salud $(54,7 \%)$. Estos datos reflejan una evidente superioridad de los porcentajes negativos sobre los positivos. En términos generales, matizar que si bien existen varias áreas que refieren buena calidad de atención en los servicios de salud pública, las percepciones negativas de la atención en salud son más elevadas que las positivas. Tras la aplicación de la prueba de Kolmogorov-Smirnov para una muestra, se encontró que las puntuaciones seguían una distribución normal, por lo que se procedió a emplear pruebas paramétricas y algunas no paramétricas, que contrastasen los resultados encontrados. A continuación se describen los resultados en función del objetivo planteado. El ANOVA de un factor indicó diferencias significativas entre las diferentes instituciones de salud estudiadas en estructura (F $3,1135=27,48$; $\mathrm{p}=0,000)$, en proceso-resultados $(F$ $3,1081=29,98 ; p=0,000)$; en accesibilidad ( $F$ 3,1173=7,19; $p=0,000)$ y en la escala total, como una dimensión de la calidad de atención en los servicios de salud pública, también demostró diferencias significativas (F $3,1026=27,94 ; p=0,000$ ). Con- 
firmándose estos resultados con la prueba no paramétrica de $\mathrm{H}$ Kruskal. Wallis: en estructura $\left(X^{2}{ }_{3}=63,79\right.$; $p=0,000)$, en proceso-resultado $\left(X^{2}\right.$ $\left.{ }_{3}=74,16 ; p=0,000\right)$, en accesibilidad $\left(X^{2}{ }_{3}=19,17 ; p=, 000\right)$ y en la escala total $\left(X^{2}{ }_{3}=67,14 ; p=0,000\right)$. Tras la aplicación de la prueba post hoc de Scheffé, se demuestran diferencias estadísticamente significativas entre las diferentes instituciones de salud pública (Hospital público, Unidades de Salud, ISSS) en comparación con las clínica y hospitales privados en función de cada una de las dimensiones de la escala de calidad de atención en los servicios de salud pública. Sin embargo, no se encontró diferencias estadísticas significativas entre los mismos centros de salud pública, en función de las escalas de calidad de atención en los servicios de salud, significando lo anterior, que en estos centros la calidad de atención es muy similar

En relación al género, la prueba $t$ demuestra que no existen diferencias significativas en estructura ( $t \quad 1,121=1,57 ; \quad p=0,12$ ), ni en accesibilidad (t $1,160=1,78 ; p=0,07$ ), pero sí existen diferencias entre hombres y mujeres en la percepción de la calidad de atención en los servicios de salud pública, en especial en la dimensión de proceso-resultado ( $t$ 1,068=2,16; $p=0.03$ ) y en la escala total ( $1,015=2,06 ; p=0,04)$; la prueba no paramétrica $U$ Mann. Whitney lo confirma, en estructura $(Z=-1,33$; $p=0,184)$, en proceso-resultado $(Z=$ $2,031 ; p=0,042)$, en accesibilidad $(Z=-$ 1,$705 ; p=0,088$ ) y en la escala total $(Z=-2,098 ; p=0,036)$. Indicando lo anterior, que las mujeres poseen una actitud más favorable que los hombres en relación a los procesos, resultados $y$ en la calidad total de los servicios de salud. EI ANOVA de un factor no demuestra diferencias significativas

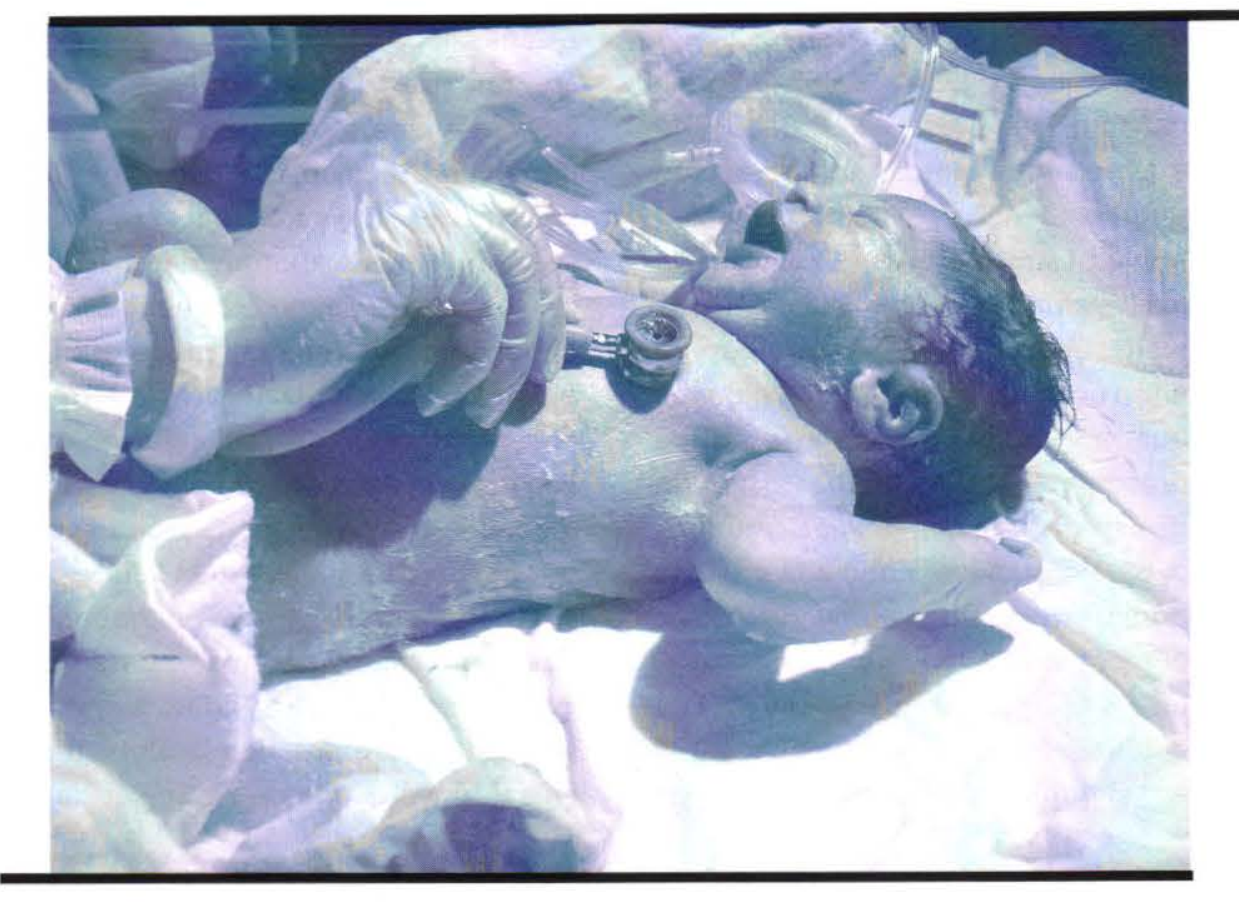

en la calidad de atención en salud pública en función del estado civil de la muestra $(F 4,1030=2,008 ; p=0,091)$, ni en relación al grado de estudio de los participantes (F 4,1019=1,312; $p=0,26$ ), tampoco por los ingresos económicos familiares (F6,985 $=0,473 ; p=0,71$ ). Por otra parte, al contrastarse la percepción de los costos de la salud en El Salvador, en función del género, se encontró ( $t$ $1,166=1,886 ; p=0,059)$ una tendencia en las mujeres a considerar más cara la atención en salud que los hombres; confirmándose lo anterior con la prueba no paramétrica de $U$ Mann Whitney ( $Z=-2,007 ; p=0,045)$. No existe correlación significativa entre la edad de la muestra y las dimensiones de la escala: estructura $(r=0,001$; $p=0,960)$, proceso-resultado $(r=0,040$; $p=0,184)$, en accesibilidad $(r=-0,034$; $p=0,246)$, en la escala total $(r=0,023 ; p$ $=0,471$ ); sin embargo, se encontraron correlaciones significativas, que van desde intermedias hasta altas, entre las escalas que conforman la prueba para evaluar la calidad de atención en los servicios de salud publica, y estas se describen de la forma siguiente: entre estructura y procesoresultado $\quad(r=0,743 ; p=0,000)$; entre estructura $y$ accesibilidad $(r=0,492 ; p=0,000)$, estructura $y$ escala total $(r=0,858 ; p=0,000)$; ente proceso-resultado y accesibilidad $(r=0,599 ; p=0,000)$ en proceso-resultado $y$ escala total ( $r=0,965 ; p=0,000)$; entreaccesibilidad $y$ escala total $(r=0,715 ; p=0,000)$.

\section{Discusión}

En el análisis descriptivo, se tienen datos $e$ indicadores llamativos relacionados con la calidad de atención médica que reciben los salvadoreños en el sistema de salud público; entresusresultadossetienen variables, que pueden ser calificadas como desfavorables o negativas (malas). Dentro de los hallazgos que presentan connotaciones negativas, se mencionan los porcentajes más sobresalientes. Estos son: la atención que recibe de la enfermera/ os, el $57,2 \%$ expresa que es mala; 
los procesos administrativos,como sacar una cita, solicitar una constancia médica, el $59,8 \%$ dicen que son muy lentos; en la atención que reciben del sistema de salud público salvadoreño, el $63,4 \%$ de los evaluados manifiestan que es inadecuada; los tiempos de espera entre una cita y otra, el $70,8 \%$ expresa que son muy largos; cuando se presenta a consulta médica el tiempo de espera para que lo vea el médico, el $69,0 \%$ manifestó que son muchas horas; en el sistema de salud público; el acceso al médico especialista, un $68,4 \%$ piensa que es difícil. Los criterios antes mencionados están en sintonía con lo planteado por

\section{Bibliografía}

- Amaya, C; (2006) La calidad de la salud, un compromiso de todos. Revista de Ciencia y Tecnología para la salud visual y ocular, No.007.

- Beaulieu, J; Scutchfield, F.D; y Kelly, A.V.(2003) Content and criterion validity evaluation of National Public Health Performance Standards measurement instruments. Public Health Rep;118: 50817.

- Beaulieu, J; y Scutchfield, F.D.(2002) Assestment of validity of the national public health performance standards: the local public health performance assessment instrument. Public Helth Rep;117:28-36.

- Barón, E.O; Dushenat, M. y Friedman, N. (2001) Evaluation of the consumer model: Relationship between patients expectations, perceptions and satisfaction with care. Int J Quality Health Care; 13(4):317-323

- Bronfman-Pertzovsky, M.N; LópezMoreno, S; Magis-Rodríguez, C; MorenoAltamirano,A;y Rutstein, R. (2003)Atención
Dueñas (2008), quien hace énfasis en las tres dimensiones de la calidad en los servicios de salud: la dimensión técnico científica con la cual se pretende garantizar servicios seguros a los usuarios dentro de la balanza riesgo beneficio; la dimensión administrativa referida a las relaciones prestadores consumidores, que se relacionan con los procesos de atención de que dispone la organización, y se hace un especial énfasis en la dimensión humana tradicionalmente desconocida y poco tratada, cuando se habla de calidad, siendo la parte fundamental de la atención en salud, ya que la razón de ser del

prenatal en el primer nivel de atención: características de los proveedores que influyen en la satisfacción de las usuarias. Salud pública de México / vol.45, no.6.

- Carretero-Dios, H; y Pérez C. (2007) Standards for the development and review of instrumental studies: Considerations about test selection in psychological research; International Journal of Clinical and Health Psychology, Vol. 7, pp. 863882

- Centre Català de la Qualitat. ISO 9001:2000 (2005) Guia de la Qualitat. Barcelona: CIDEM; 2001. 331 Guix Oliver J. Calidad en salud pública Gac Sanit. 19(4):

- Comisión de Derechos Humanos de EI Salvador. (2007) Análisis de las condiciones de salud en EI Salvador. (Recuperado 3 de febrero del 2009) de http://wnww.bvsde. paho.org/bvsacd/cd53/dersal/cap6.pdf

- Consultora en Marketing, Recursos Humanos y Servicios en Informática, Capacitación Laboral y Empresarial, (2008). Estrategia Magazine (Recuperado el 20/04/2009). Disponible en http:// www.gestiopolis.com/administracion- servicio de salud es el ser humano, como un ser biopsicosocial. De igual forma, Christofides, Jewkes, Webster, Kekana, Abrahams y Martín (2005) encontró (en tres provincias de Sur África) que el sistema de salud era profundamente defectuoso, con problemas de inaccesibilidad, con prejuicio y con falta de entrenamiento en todos los niveles (Human Rights Watch 1997). Se encontró a menudo largas esperas para recibir los servicios de salud, de uno a cinco pacientes esperando más de cinco horas para que lo viera un cirujano del distrito. (Swart, Gilchrist, Butchard, Seedat y Martín, 1999).

estrategia/estrategia/calidad-en-laatencion-al-cliente-1.htm

- Chan, M. (2008) Informe sobre salud en el mundo. Directora General, Organización Mundial de la Salud.

- Cebrián, S. (1995) Glosario de economía de la salud, PALTEX. Organización Panamericana de la Salud. España.

- Consultora en Marketing, Recursos Humanos y Servicios en Informática, Capacitación

- Laboral y Empresarial, (2008). Estrategia Magazine (Recuperado el 20/04/2009). Disponible http://www.gestiopolis.com/ administracion-estrategia/estrategia/ calidad-en-la-atencion-al-cliente-1.htm

- Drain M. (2001) Quality improvement in primary care and the importance of patients perceptions. J Ambulatory Care Manag ;24(2):30-46.

- Draper, M; Cohen, P. y Buchan, H. (2001) Seeking consumer views: What use results of hospital patient satisfaction surveys? Int J Quality Health Care;13(6):463-468. 